\title{
A New Approach to Fuzzy Differential Equations Using Weakly- Compatible Self-Mappings in Fuzzy Metric Spaces
}

\author{
Iqra Shamas, ${ }^{1}$ Saif Ur Rehman $\mathbb{D}^{1},{ }^{1}$ Naeem Jan ${ }^{(D)},{ }^{1}$ Abdu Gumaei ${ }^{(D)},{ }^{2}$ \\ and Mabrook Al-Rakhami ${ }^{3}{ }^{3}$ \\ ${ }^{1}$ Department of Mathematics, Gomal University, Dera Ismail Khan 29050, Pakistan \\ ${ }^{2}$ Computer Science Department, Faculty of Applied Sciences, Taiz University, Taiz 6803, Yemen \\ ${ }^{3}$ STC's Artificial Intelligence Chair, Department of Information Systems, College of Computer and Information Sciences, \\ King Saud University, Riyadh 11543, Saudi Arabia
}

Correspondence should be addressed to Mabrook Al-Rakhami; malrakhami@ksu.edu.sa

Received 27 June 2021; Revised 28 September 2021; Accepted 29 September 2021; Published 12 October 2021

Academic Editor: Douadi Drihem

Copyright (c) 2021 Iqra Shamas et al. This is an open access article distributed under the Creative Commons Attribution License, which permits unrestricted use, distribution, and reproduction in any medium, provided the original work is properly cited.

The key objective of this research article includes the study of some rational type coincidence point and deriving common fixed point (CFP) results for rational type weakly-compatible three self-mappings in fuzzy metric (FM) space. The "triangular property of FM" is used as a fundamental tool. Moreover, some unique coincidence points and CFP theorems were presented for three selfmappings in an FM space under the conditions of rational type weakly-compatible fuzzy-contraction. In addition, some suitable examples are also given. Furthermore, an application of fuzzy differential equations is provided in the aid of the proposed work. Hence, the innovative direction of rational type weakly-compatible fuzzy-contraction with the application of fuzzy differential equations in FM space will certainly play a vital role in the related fields. It has the potential to be extended in any direction with different types of weakly-compatible fuzzy-contraction conditions for self-mappings with different types of differential equations.

\section{Introduction}

In 1922, Banach [1] proved a "Banach contraction principle for fixed point (FP)" which is stated as "A self-mapping in a complete metric space satisfy the contraction condition has a unique FP." After the publication of the "Banach contraction principle," many researchers contributed in flourishing the FP theory. Thus, different types of contractive results were established for FP and CFP for different types of mappings in the context of metric spaces. Kannan [2], Chatterjea [3, 4], Ali et al. [5], Covitz and Nadler [6], Altun et al. [7], Khan [8], Rehman et al. [9], and Sahin [10] proved some singlevalued and multivalued contractive type FP, CFP, and best proximity point results in different types of spaces.

In 1965, the concept of fuzzy sets was given by Zadeh [11], and this concept is investigated, used, and applied in many directions. In [12], Kramosil and Michalek used the concept of fuzzy sets together with metric space and intro- duced the notion of FM space and some more notions. In 1988, Grabiec [13] used the concept of Kramosil and Michalek [12] and proved two FP theorems of "Banach and Edelstein contraction mapping theorems on complete and compact fuzzy metric spaces, respectively." Later on, George and Veeramani [14] presented the stronger form of metric fuzziness. In 2002, Gregory and Sapena [15] proved some contractive type FP theorems in complete FM spaces in the sense of $[12,14]$. In [16], Sadeghi et al. extended and improved the result of Gregory and Sapena [15] and proved some FP and coincidence point theorems by using an implicit relation for set-valued-mappings on complete partially ordered FM spaces. Bari and Vetro [17] established some FP results by using attractors and weak-fuzzy contractive mappings in FM space. While Hadzic and Pap [18] proved "a FP theorem for multi-valued mappings in probabilistic metric spaces with an application in FM spaces." Imdad and Ali [19], Shamas et al. [20], Som [21], and Pant 
and Chauhan [22] proved FP and CFP theorems for different contractive type mappings in FM spaces. Saleh et al. $[23,24]$ established different contractive type FP results in FM spaces. Recently, Rehman et al. [25] introduced the rational type fuzzy-contraction condition in FM spaces and proved some FP theorems with an application. In 2015, Oner et al. [26] introduced the notion of fuzzy cone metric (FCM) space. They proved some basic properties and a "fuzzy cone Banach contraction theorem" for FP with the assumption that "the fuzzy cone contractive sequences are Cauchy." Later on, Rehman and Li [27] established some FP theorems in FCM space without the assumption that the "fuzzy cone contractive sequences are Cauchy." Jabeen et al. [28] proved some common FP results in FCM spaces by using the contractive type weakly-compatible self-mappings with an application. By using the concept of [27], Rehman and Aydi [29] proved some rational type CFP theorems in FCM spaces with an application to Fredholm integral equations.

The chief purpose behind this article is the introduction of a new concept of rational type weakly-compatible fuzzycontraction maps for three self-mappings in FM spaces. We used the "triangular property of fuzzy metric" as an elementary tool and proved some unique coincidence points and CFP theorems under the rational type weakly compatible fuzzy-contraction conditions for three self-mappings in FM spaces with some suitable examples. Moreover, we applied the fuzzy differential equations for a unique solution in order to support our study. As a result, the current novel direction of rational type weakly-compatible fuzzycontraction with the application of fuzzy differential equations in FM space will play a vital role in the related fields. It has the potential to be extended in any direction with different types of weakly-contraction conditions for selfmappings with different types of differential equations.

This paper is organized as follows: Section 2 named as preliminaries consists of the basic concepts related to our main work. In Section 3, we established some coincidence points and CFP theorems under the rational type weaklycompatible fuzzy-contraction conditions for three selfmappings in FM spaces with examples to verify the validity of our work. Section 4 deals with the application of the fuzzy differential equations to support our work. Finally, the last section, Section 5 concludes this article.

\section{Preliminaries}

In this section, we present some preliminaries related to our main work such as continuous $t$-norm, FM space, Cauchy sequence, complete FM space, fuzzy-contraction, fuzzycontractive sequence, and weakly-compatible self-mappings.

Definition 1 (see [30]). An operation $*:[0,1]^{2} \longrightarrow[0,1]$ is called a continuous $t$-norm, if

(i) * continuous

(ii) $*$ is commutative and associative

(iii) $1 * \zeta_{1}=\zeta_{1}$ and $\zeta_{1} * \zeta_{2} \leq \zeta_{3} * \zeta_{4}$, whenever $\zeta_{1} \leq \zeta_{3}$ and $\zeta_{2} \leq \zeta_{4}, \forall \zeta_{1}, \zeta_{2}, \zeta_{3}, \zeta_{4} \in[0,1]$
The basic continuous $t$-norms of minimum, product, and Lukasiewicz are defined by Schweizer and Sklar [30], respectively, as follows

(i) The minimum $t$-norm is $\zeta_{1} * \zeta_{2}=\min \left\{\zeta_{1}, \zeta_{2}\right\}$

(ii) The product $t$-norm is $\zeta_{1} * \zeta_{2}=\zeta_{1} \zeta_{2}$

(iii) The Lukasiewicz $t$-norm is $\zeta_{1} * \zeta_{2}=\max \left\{\zeta_{1}+\zeta_{2}\right.$ $-1,0\}$

Definition 2 (see [14]). A 3-tuple $\left(W, \mathbb{M}_{r}, *\right)$ is said to be a FM space if $W$ is an arbitrary set, $*$ is a continuous $t$ -norm and $\mathbb{M}_{r}$ is a fuzzy set on $W^{2} \times(0, \infty)$ satisfying the following conditions:

(i) $\mathbb{M}_{r}(w, x, t)>0$

(ii) $\mathbb{M}_{r}(w, x, t)=1 \Leftrightarrow w=x$

(iii) $\mathbb{M}_{r}(w, x, t)=\mathbb{M}_{r}(x, w, t)$

(iv) $\mathbb{M}_{r}(w, \rho, t) * \mathbb{M}_{r}(\rho, x, s) \leq \mathbb{M}_{r}(w, x, t+s)$

(v) $\mathbb{M}_{r}(w, x,):.(0, \infty) \longrightarrow[0,1]$ is continuous

$$
\forall w, x, \rho \in W \text { and } t, s \in(0, \infty) \text {. }
$$

Lemma 3 (see [14]). $\mathbb{M}_{r}(w, x, *)$ is nondecreasing $\forall w, x \in W$.

Definition 4 (see $[14,15])$. Let $\left(W, \mathbb{M}_{r}, *\right)$ be a FM space, $w$ $\in W$ and $\left(w_{j}\right)$ is a sequence in $W$. Then,

(i) $\left(w_{j}\right)$ converges to $w$ if $\rho \in(0,1)$ and $t>0 \exists j_{1} \in \mathbb{N}$, such that $\mathbb{M}_{r}\left(w_{j}, w, t\right)>1-\rho, \forall j \geq j_{1}$. We may write this $\lim _{j \longrightarrow \infty} w_{j}=w$ or $w_{j} \longrightarrow w$ as $j \longrightarrow \infty$

(ii) $\left(w_{j}\right)$ is a Cauchy sequence if $\rho \in(0,1)$ and $t>0$ $\exists j_{1} \in \mathbb{N}$ such that $\mathbb{M}_{r}\left(w_{j}, w_{k}, t\right)>1-\rho, \forall j, k \geq j_{1}$

(iii) $\left(W, \mathbb{M}_{r}, *\right)$ is complete if every Cauchy sequence is convergent in $W$

(iv) Fuzzy-contractive if $\exists a \in(0,1)$ and satisfying

$\frac{1}{\mathbb{M}_{r}\left(w_{j}, w_{j+1}, t\right)}-1 \leq a\left(\frac{1}{\mathbb{M}_{r}\left(w_{j-1}, w_{j}, t\right)}-1\right)$ for $t>0, j \geq 1$.

Throughout this paper, $\mathbb{N}$ represents the set of natural numbers.

Lemma 5 (see [14]). Let $\left(W, \mathbb{M}_{r}, *\right)$ be a FM space. $A$ sequence $\left(w_{j}\right)$ in $W$ converges to $w \in W$ if and only if $\mathbb{M}_{r}$ $\left(w_{j}, w, t\right) \longrightarrow 1$, as $j \longrightarrow \infty$, for $t>0$. 
Definition 6 (see [17]). Let $\left(W, \mathbb{M}_{r}, *\right)$ be a FM space. The fuzzy metric $\mathbb{M}_{r}$ is triangular, if

$$
\begin{aligned}
\frac{1}{\mathbb{M}_{r}(w, x, t)}-1 \leq & \left(\frac{1}{\mathbb{M}_{r}(w, \rho, t)}-1\right) \\
& +\left(\frac{1}{\mathbb{M}_{r}(\rho, x, t)}-1\right) \forall w, \rho, x \in W, t>0 .
\end{aligned}
$$

Definition 7 (see [15]). Let $\left(W, \mathbb{M}_{r}, *\right)$ be a FM space. A mapping $A: W \longrightarrow W$ is said to be a fuzzy-contractive if $\exists a \epsilon$ $(0,1)$ such that

$$
\frac{1}{\mathbb{M}_{r}(A w, A x, t)}-1 \leq a\left(\frac{1}{\mathbb{M}_{r}(w, x, t)}-1\right) \forall w, x \in W, t>0 .
$$

Definition 8 (see [31]). Let $A$ and $\ell$ be two self-mappings on a nonempty set $W$ (i.e., $A, \ell: W \longrightarrow W$ ). If there exists $u$ $\in W$ and $u=A v=\ell v$ for some $v \in W$. Then, $v$ is called a coincidence point of $A$ and $\ell$, and $u$ is called a point of coincidence of the mappings $A$ and $\ell$. The mappings $A$ and $\ell$ are said to be weakly-compatible if they commute at their coincidence point, i.e., $A v=\ell v$ for some $v \in W$, then $A \ell v=\ell A v$.

Proposition 9 (see [31]). Let $A$ and $\ell$ be weakly-compatible self-mappings on a nonempty set $W$. If $A$ and $\ell$ have a unique point of coincidence such that $u=A v=\ell v$, then, $u$ is known as the unique common FP of $A$ and $\ell$.

\section{Main Result}

This section deals with the main results of our paper, here, we establish some coincidence point and CFP theorems under the rational type weakly-compatible fuzzycontractive for three self-mappings in FM spaces with some suitable examples. Throughout main results, we use the concept of a binary operation $*$ is a continuous product $t$-norm which is defined as:

$$
\xi * \zeta=\xi \cdot \zeta \text { for all } \xi, \zeta \in[0,1]
$$

Now we are in the position to present our first main result.

Theorem 10. Let a fuzzy metric $\mathbb{M}_{r}$ is triangular in a complete FM space $\left(W, \mathbb{M}_{r}, *\right)$ and let $A, B, \ell: W \longrightarrow W$ be three self-mappings, satisfies for all $w, x \in W$,

$$
\begin{aligned}
\frac{1}{\mathbb{M}_{r}(A w, B x, t)}-1 \leq & a\left(\frac{1}{\mathbb{M}_{r}(\ell w, \ell x, t)}-1\right) \\
& +b\left(\frac{\mathbb{M}_{r}(\ell w, \ell x, t)}{\mathbb{M}_{r}(\ell x, A w, 2 t) \cdot \mathbb{M}_{r}(\ell w, B x, 2 t)}-1\right) \\
& +c\left(\frac{1}{\mathbb{M}_{r}(\ell w, A w, t)}-1+\frac{1}{\mathbb{M}_{r}(\ell x, B x, t)}-1\right),
\end{aligned}
$$

for $t>0$ and $0 \leq a, b, c<1$ with $(a+b+2 c)<1$. If $A(W) \cup$ $B(W) \subset \ell(W)$, where $\ell(W)$ is a complete subspace of $W$. Then $A, B$, and $\ell$ have a unique point of coincidence. Moreover, if the pairs $(A, \ell)$ and $(B, \ell)$ are weakly compatible. Then, $A, B$, and $\ell$ have a unique CFP in $W$.

Proof. Let $w_{0}$ be the arbitrary point of $W$. Using the condition $A(W) \cup B(W) \subset \ell(W)$ choose a sequence $\left(w_{i}\right)$ in $W$ such that

$$
\ell w_{2 i+1}=A w_{2 i} \text { and } \ell w_{2 i+2}=B w_{2 i+1} \text {, for all } i \geq 0
$$

Now, by (6), for $t>0$,

$$
\begin{aligned}
& \frac{1}{\mathbb{M}_{r}\left(\ell w_{2 i+1}, \ell w_{2 i+2}, t\right)}-1=\frac{1}{\mathbb{M}_{r}\left(A w_{2 i}, B w_{2 i+1}, t\right)}-1 \\
& \leq a\left(\frac{1}{\mathbb{M}_{r}\left(\ell w_{2 i}, \ell w_{2 i+1}, t\right)}-1\right) \\
&+b\left(\frac{\mathbb{M}_{r}\left(\ell w_{2 i}, \ell w_{2 i+1}, t\right)}{\mathbb{M}_{r}\left(\ell w_{2 i+1}, A w_{2 i}, 2 t\right) \cdot \mathbb{M}_{r}\left(\ell w_{2 i}, B w_{2 i+1}, 2 t\right)}-1\right) \\
&+c\left(\frac{1}{\mathbb{M}_{r}\left(\ell w_{2 i}, A w_{2 i}, t\right)}-1+\frac{1}{\mathbb{M}_{r}\left(\ell w_{2 i+1}, B w_{2 i+1}, t\right)}-1\right) \\
&=a\left(\frac{1}{\mathbb{M}_{r}\left(\ell w_{2 i}, \ell w_{2 i+1}, t\right)}-1\right) \\
&+b\left(\frac{\mathbb{M}_{r}\left(\ell w_{2 i}, \ell w_{2 i+1}, t\right)}{\mathbb{M}_{r}\left(\ell w_{2 i+1}, \ell w_{2 i+1}, 2 t\right) \cdot \mathbb{M}_{r}\left(\ell w_{2 i}, \ell w_{2 i+2}, 2 t\right)}-1\right) \\
&+c\left(\frac{1}{M_{r}\left(\ell w_{2 i}, \ell w_{2 i+1}, t\right)}-1+\frac{1}{M_{r}\left(\ell w_{2 i+1}, \ell w_{2 i+2}, t\right)}-1\right) \\
&=a\left(\frac{1}{\mathbb{M}_{r}\left(\ell w_{2 i}, \ell w_{2 i+1}, t\right)}-1\right)+b\left(\frac{\mathbb{M}_{r}\left(\ell w_{2 i}, \ell w_{2 i+1}, t\right)}{\mathbb{M}_{r}\left(\ell w_{2 i}, \ell w_{2 i+2}, 2 t\right)}-1\right) \\
&+ c\left(\frac{1}{\mathbb{M}_{r}\left(\ell w_{2 i}, \ell w_{2 i+1}, t\right)}-1+\frac{1}{\mathbb{M}_{r}\left(\ell w_{2 i+1}, \ell w_{2 i+2}, t\right)}-1\right)
\end{aligned}
$$

Now by using Definition 2 (iv), $\mathbb{M}_{r}\left(\ell w_{2 i}, \ell w_{2 i+2}, 2 t\right) \geq \mathbb{M}_{r}$ $\left(\ell w_{2 i}, \ell w_{2 i+1}, t\right) \cdot \mathbb{M}_{r}\left(\ell w_{2 i+1}, \ell w_{2 i+2}, t\right)$ for $t>0$,

$$
\begin{aligned}
\frac{1}{\mathbb{M}_{r}\left(\ell w_{2 i+1}, \ell w_{2 i+2}, t\right)}-1 \leq a\left(\frac{1}{\mathbb{M}_{r}\left(\ell w_{2 i}, \ell w_{2 i+1}, t\right)}-1\right) \\
\quad+b\left(\frac{\mathbb{M}_{r}\left(\ell w_{2 i}, \ell w_{2 i+1}, t\right)}{\mathbb{M}_{r}\left(\ell w_{2 i}, \ell w_{2 i+1}, t\right) \cdot \mathbb{M}_{r}\left(\ell w_{2 i+1}, \ell w_{2 i+2}, t\right)}-1\right) \\
\quad+c\left(\frac{1}{\mathbb{M}_{r}\left(\ell w_{2 i}, \ell w_{2 i+1}, t\right)}-1+\frac{1}{\mathbb{M}_{r}\left(\ell w_{2 i+1}, \ell w_{2 i+2}, t\right)}-1\right) \\
=a\left(\frac{1}{\mathbb{M}_{r}\left(\ell w_{2 i}, \ell w_{2 i+1}, t\right)}-1\right)+b\left(\frac{1}{\mathbb{M}_{r}\left(\ell w_{2 i+1}, \ell w_{2 i+2}, t\right)}-1\right) \\
\quad+c\left(\frac{1}{\mathbb{M}_{r}\left(\ell w_{2 i}, \ell w_{2 i+1}, t\right)}-1+\frac{1}{\mathbb{M}_{r}\left(\ell w_{2 i+1}, \ell w_{2 i+2}, t\right)}-1\right) \\
=(a+c)\left(\frac{1}{\mathbb{M}_{r}\left(\ell w_{2 i}, \ell w_{2 i+1}, t\right)}-1\right)+(b+c)\left(\frac{1}{\mathbb{M}_{r}\left(\ell w_{2 i+1}, \ell w_{2 i+2}, t\right)}-1\right) .
\end{aligned}
$$

After simplification, we obtain

$\frac{1}{\mathbb{M}_{r}\left(\ell w_{2 i+1}, \ell w_{2 i+2}, t\right)}-1 \leq Y\left(\frac{1}{\mathbb{M}_{r}\left(\ell w_{2 i}, \ell w_{2 i+1}, t\right)}-1\right)$ for $t>0$, 
where $Y=(a+c) /(1-b-c)<1$. Similarly, again by the view of (6), for $t>0$,

$$
\begin{aligned}
& \frac{1}{\mathbb{M}_{r}\left(\ell w_{2 i+2}, \ell w_{2 i+3}, t\right)}-1=\frac{1}{\mathbb{M}_{r}\left(A w_{2 i+2}, B w_{2 i+1}, t\right)}-1 \\
& \leq a\left(\frac{1}{\mathbb{M}_{r}\left(\ell w_{2 i+2}, \ell w_{2 i+1}, t\right)}-1\right) \\
&+b\left(\frac{\mathbb{M}_{r}\left(\ell w_{2 i+2}, \ell w_{2 i+1}, t\right)}{\mathbb{M}_{r}\left(\ell w_{2 i+1}, A w_{2 i+2}, 2 t\right) \cdot \mathbb{M}_{r}\left(\ell w_{2 i+2}, B w_{2 i+1}, 2 t\right)}-1\right) \\
&+c\left(\frac{1}{\mathbb{M}_{r}\left(\ell w_{2 i+2}, A w_{2 i+2}, t\right)}-1+\frac{1}{\mathbb{M}_{r}\left(\ell w_{2 i+1}, B w_{2 i+1}, t\right)}-1\right) \\
&= a\left(\frac{1}{\mathbb{M}_{r}\left(\ell w_{2 i+2}, \ell w_{2 i+1}, t\right)}-1\right) \\
&+b\left(\frac{\mathbb{M}_{r}\left(\ell w_{2 i+2}, \ell w_{2 i+1}, t\right)}{\mathbb{M}_{r}\left(\ell w_{2 i+1}, \ell w_{2 i+3}, 2 t\right) \cdot \mathbb{M}_{r}\left(\ell w_{2 i+2}, \ell w_{2 i+2}, 2 t\right)}-1\right) \\
&+c\left(\frac{1}{\mathbb{M}_{r}\left(\ell w_{2 i+2}, \ell w_{2 i+3}, t\right)}-1+\frac{1}{\mathbb{M}_{r}\left(\ell w_{2 i+1}, \ell w_{2 i+2}, t\right)}-1\right) \\
&= a\left(\frac{1}{\mathbb{M}_{r}\left(\ell w_{2 i+2}, \ell w_{2 i+1}, t\right)}-1\right)+b\left(\frac{\mathbb{M}_{r}\left(\ell w_{2 i+2}, \ell w_{2 i+1}, t\right)}{\mathbb{M}_{r}\left(\ell w_{2 i+1}, \ell w_{2 i+3}, 2 t\right)}-1\right) \\
&+c\left(\frac{1}{\mathbb{M}_{r}\left(\ell w_{2 i+2}, \ell w_{2 i+3}, t\right)}-1+\frac{1}{\mathbb{M}_{r}\left(\ell w_{2 i+1}, \ell w_{2 i+2}, t\right)}-1\right) .
\end{aligned}
$$

Now by using Definition 2 (iv), $\mathbb{M}_{r}\left(\ell w_{2 i+1}, \ell w_{2 i+3}, 2 t\right) \geq$ $\mathbb{M}_{r}\left(\ell w_{2 i+1}, \ell w_{2 i+2}, t\right) \cdot \mathbb{M}_{r}\left(\ell w_{2 i+2}, \ell w_{2 i+3}, t\right)$ for $t>0$,

$$
\begin{aligned}
& \frac{1}{\mathbb{M}_{r}\left(\ell w_{2 i+2}, \ell w_{2 i+3}, t\right)}-1 \leq a\left(\frac{1}{\mathbb{M}_{r}\left(\ell w_{2 i+2}, \ell w_{2 i+1}, t\right)}-1\right) \\
& +b\left(\frac{\mathbb{M}_{r}\left(\ell w_{2 i+2}, \ell w_{2 i+1}, t\right)}{\mathbb{M}_{r}\left(\ell w_{2 i+1}, \ell w_{2 i+2}, t\right) \cdot \mathbb{M}_{r}\left(\ell w_{2 i+2}, \ell w_{2 i+3}, t\right)}-1\right) \\
& \quad+c\left(\frac{1}{\mathbb{M}_{r}\left(\ell w_{2 i+2}, \ell w_{2 i+3}, t\right)}-1+\frac{1}{\mathbb{M}_{r}\left(\ell w_{2 i+1}, \ell w_{2 i+2}, t\right)}-1\right) \\
& =a\left(\frac{1}{\mathbb{M}_{r}\left(\ell w_{2 i+2}, \ell w_{2 i+1}, t\right)}-1\right)+b\left(\frac{1}{\mathbb{M}_{r}\left(\ell w_{2 i+2}, \ell w_{2 i+3}, t\right)}-1\right) \\
& \quad+c\left(\frac{1}{\mathbb{M}_{r}\left(\ell w_{2 i+2}, \ell w_{2 i+3}, t\right)}-1+\frac{1}{\mathbb{M}_{r}\left(\ell w_{2 i+1}, \ell w_{2 i+2}, t\right)}-1\right) \\
& =(a+c)\left(\frac{1}{\mathbb{M}_{r}\left(\ell w_{2 i+1}, \ell w_{2 i+2}, t\right)}-1\right)+(b+c) \\
& \quad \cdot\left(\frac{1}{\mathbb{M}_{r}\left(\ell w_{2 i+2}, \ell w_{2 i+3}, t\right)}-1\right) .
\end{aligned}
$$

After simplification, we obtain

$\frac{1}{\mathbb{M}_{r}\left(\ell w_{2 i+2}, \ell w_{2 i+3}, t\right)}-1 \leq Y\left(\frac{1}{\mathbb{M}_{r}\left(\ell w_{2 i+1}, \ell w_{2 i+2}, t\right)}-1\right)$ for $t>0$

where $Y$ value is same as in (10). Now from (10), (13), and by induction,

$$
\begin{aligned}
& \frac{1}{\mathbb{M}_{r}\left(\ell w_{2 i+2}, \ell w_{2 i+3}, t\right)}-1 \leq Y\left(\frac{1}{\mathbb{M}_{r}\left(\ell w_{2 i+1}, \ell w_{2 i+2}, t\right)}-1\right) \\
& \quad \leq Y^{2}\left(\frac{1}{\mathbb{M}_{r}\left(\ell w_{2 i}, \ell w_{2 i+1}, t\right)}-1\right) \\
& \quad \leq \cdots \leq Y^{2 i+2}\left(\frac{1}{\mathbb{M}_{r}\left(\ell w_{0}, \ell w_{1}, t\right)}-1\right) \longrightarrow 0 \text {, as } i \longrightarrow \infty
\end{aligned}
$$

Hence, $\left(\ell w_{i}\right)_{i \geq 0}$ is a fuzzy-contractive sequence in $(W$, $\left.\mathbb{M}_{r}, *\right)$, therefore,

$$
\lim _{i \longrightarrow \infty} \mathbb{M}_{r}\left(\ell w_{i}, \ell w_{i-1}, t\right)=1 \text { for } t>0 .
$$

Since $\mathbb{M}_{r}$ is triangular, $j>i>i_{0}$,

$$
\begin{aligned}
& \frac{1}{\mathbb{M}_{r}\left(\ell w_{i}, \ell w_{j}, t\right)}-1 \leq\left(\frac{1}{\mathbb{M}_{r}\left(\ell w_{i}, \ell w_{i+1}, t\right)}-1\right) \\
& \quad+\left(\frac{1}{\mathbb{M}_{r}\left(\ell w_{i+1}, \ell w_{i+2}, t\right)}-1\right)+\cdots+\left(\frac{1}{\mathbb{M}_{r}\left(\ell w_{i-1}, \ell w_{j}, t\right)}-1\right) \\
& \leq Y^{i}\left(\frac{1}{\mathbb{M}_{r}\left(\ell w_{i}, \ell w_{i+1}, t\right)}-1\right)+Y^{i+1}\left(\frac{1}{\mathbb{M}_{r}\left(\ell w_{i+1}, \ell w_{i+2}, t\right)}-1\right) \\
& \quad+\cdots+Y^{j-1}\left(\frac{1}{\mathbb{M}_{r}\left(\ell w_{i-1}, \ell w_{j}, t\right)}-1\right) \leq\left(Y^{i}+Y^{i+1}+\cdots+Y^{j-1}\right) \\
& \quad \cdot\left(\frac{1}{\mathbb{M}_{r}\left(\ell w_{0}, \ell w_{1}, t\right)}-1\right) \leq\left(\frac{Y^{i}}{1-Y}\right)\left(\frac{1}{\mathbb{M}_{r}\left(\ell w_{0}, \ell w_{1}, t\right)}-1\right) \\
& \longrightarrow 0, \text { as } i \longrightarrow \infty .
\end{aligned}
$$

This shows that $\left(\ell w_{i}\right)$ is a Cauchy sequence, and $\ell(W)$ is a complete subspace of $W$. Hence, $\exists u, v \in W$ such that $\ell w_{i}$ $\longrightarrow u=\ell v$ as $i \longrightarrow \infty$, i.e.,

$$
\lim _{i \longrightarrow \infty} \mathbb{M}_{r}\left(u, \ell w_{i}, t\right)=\mathbb{M}_{r}(u, \ell v, t)=1 \text { for } t>0 .
$$

Since $\mathbb{M}_{r}$ is triangular,

$$
\begin{aligned}
\frac{1}{\mathbb{M}_{r}(\ell v, A v, t)}-1 \leq & \left(\frac{1}{\mathbb{M}_{r}\left(\ell v, \ell w_{2 i+2}, t\right)}-1\right) \\
& +\left(\frac{1}{\mathbb{M}_{r}\left(\ell w_{2 i+2}, A v, t\right)}-1\right) \text { for } t>0 .
\end{aligned}
$$

Now from (6), (15), (17), and by using Definition 2 (iv), for $t>0$, 


$$
\begin{aligned}
& \frac{1}{\mathbb{M}_{r}\left(\ell w_{2 i+2}, A v, t\right)}-1=\left(\frac{1}{\mathbb{M}_{r}\left(A v, B w_{2 i+1}, t\right)}-1\right) \\
& \leq a\left(\frac{1}{\mathbb{M}_{r}\left(\ell v, \ell w_{2 i+1}, t\right)}-1\right) \\
& \quad+b\left(\frac{\mathbb{M}_{r}\left(\ell v, \ell w_{2 i+1}, t\right)}{\mathbb{M}_{r}\left(\ell w_{2 i+1}, A v, 2 t\right) \cdot \mathbb{M}_{r}\left(\ell v, B w_{2 i+1}, 2 t\right)}-1\right) \\
& \quad+c\left(\frac{1}{\mathbb{M}_{r}(\ell v, A v, t)}-1+\frac{1}{\mathbb{M}_{r}\left(\ell w_{2 i+1}, B w_{2 i+1}, t\right)}-1\right) \\
& \leq a\left(\frac{1}{\mathbb{M}_{r}\left(\ell v, \ell w_{2 i+1}, t\right)}-1\right) \\
&+b\left(\frac{\mathbb{M}_{r}\left(\ell v, \ell w_{2 i+1}, t\right)}{\left.\mathbb{M}_{r}\left(\ell w_{2 i+1}, \ell v, t\right) \cdot \mathbb{M}_{r}, \ell v, A v, t\right) \cdot \mathbb{M}_{r}\left(\ell v, \ell w_{2 i+2}, 2 t\right)}-1\right) \\
&+c\left(\frac{1}{\mathbb{M}_{r}(\ell v, A v, t)}-1+\frac{1}{\mathbb{M}_{r}\left(\ell w_{2 i+1}, \ell w_{2 i+2}, t\right)}-1\right) \\
& \longrightarrow(b+c)\left(\frac{1}{\mathbb{M}_{r}(\ell v, A v, t)}-1\right) \text { as } i \longrightarrow \infty .
\end{aligned}
$$

Then,

$$
\begin{aligned}
& \underset{i \longrightarrow \infty}{\limsup }\left(\frac{1}{\mathbb{M}_{r}\left(\ell w_{2 i+2}, A v, t\right)}-1\right) \\
& \quad \leq(b+c)\left(\frac{1}{\mathbb{M}_{r}(\ell v, A v, t)}-1\right) \text { for } t>0 .
\end{aligned}
$$

Now, from (17), (18), and (20), we obtain

$$
\frac{1}{\mathbb{M}_{r}(\ell v, A v, t)}-1 \leq(b+c)\left(\frac{1}{\mathbb{M}_{r}(\ell v, A v, t)}-1\right) \text { for } t>0 .
$$

Notice that $(b+c)<1$, where $(a+b+2 c)<1$, therefore, $\mathbb{M}_{r}(\ell v, A v, t)=\mathbb{M}_{r}(u, A v, t)=1 \Rightarrow u=\ell v=A v$ for $t>0$. Next, we have to prove that $u=\ell v=B v$. Since, $\mathbb{M}_{r}$ is triangular,

$$
\begin{aligned}
\frac{1}{\mathbb{M}_{r}(\ell v, B v, t)}-1 \leq & \left(\frac{1}{\mathbb{M}_{r}\left(\ell v, \ell w_{2 i+1}, t\right)}-1\right) \\
& +\left(\frac{1}{\mathbb{M}_{r}\left(\ell w_{2 i+1}, B v, t\right)}-1\right) \text { for } t>0 .
\end{aligned}
$$

Now, again from (6), (15), (17), and by using Definition 2 (iv), for $t>0$,

$$
\begin{aligned}
& \frac{1}{\mathbb{M}_{r}\left(\ell w_{2 i+1}, B v, t\right)}-1=\left(\frac{1}{\mathbb{M}_{r}\left(A w_{2 i}, B v, t\right)}-1\right) \leq a\left(\frac{1}{\mathbb{M}_{r}\left(\ell w_{2 i}, \ell v, t\right)}-1\right) \\
& \quad+b\left(\frac{\mathbb{M}_{r}\left(\ell w_{2 i}, \ell v, t\right)}{\mathbb{M}_{r}\left(\ell v, A w_{2 i}, 2 t\right) \cdot \mathbb{M}_{r}\left(\ell w_{2 i}, B v, 2 t\right)}-1\right) \\
& \quad+c\left(\frac{1}{\mathbb{M}_{r}\left(\ell w_{2 i}, A w_{2 i}, t\right)}-1+\frac{1}{\mathbb{M}_{r}(\ell v, B v, t)}-1\right) \\
& \leq a\left(\frac{1}{\mathbb{M}_{r}\left(\ell w_{2 i}, \ell v, t\right)}-1\right) \\
& \quad+b\left(\frac{\mathbb{M}_{r}\left(\ell w_{2 i}, \ell v, t\right)}{\mathbb{M}_{r}\left(\ell v, \ell w_{2 i+1}, 2 t\right) \cdot \mathbb{M}_{r}\left(\ell w_{2 i}, \ell v, t\right) \cdot \mathbb{M}_{r}(\ell v, B v, t)}-1\right) \\
& \quad+c\left(\frac{1}{\mathbb{M}_{r}\left(\ell w_{2 i}, \ell w_{2 i+1}, t\right)}-1+\frac{1}{\mathbb{M}_{r}(\ell v, B v, t)}-1\right) .
\end{aligned}
$$

Then,

$$
\limsup _{i \rightarrow \infty}\left(\frac{1}{\mathbb{M}_{r}\left(\ell w_{2 i+1}, B v, t\right)}-1\right) \leq(b+c)\left(\frac{1}{\mathbb{M}_{r}(\ell v, B v, t)}-1\right) \text { for } t>0 .
$$

Now, from (17), (22), and (24), we obtain

$$
\frac{1}{\mathbb{M}_{r}(\ell v, B v, t)}-1 \leq(b+c)\left(\frac{1}{\mathbb{M}_{r}(\ell v, B v, t)}-1\right) \text { for } t>0 .
$$

Note that $(b+c)<1$, where $(a+b+2 c)<1$, therefore, $\mathbb{M}_{r}(\ell v, B v, t)=\mathbb{M}_{r}(u, B v, t)=1 \Rightarrow u=\ell v=A v$ for $t>0$. Hence, $u$ is a common coincidence point of the mappings $\ell$, $A$ and $B$ in $W$ such that $u=\ell v=A v=B v$.

Next, we prove the uniqueness of a coincidence point in $\left(W, \mathbb{M}_{r}, *\right)$ for the mappings $\ell, A$ and $B$. Let $u^{*}$ be the other common coincidence point in $W$ such that $u^{*}=\ell v^{*}=A v^{*}$ $=B v^{*}$ for some $v^{*} \in W$. Then, from (6) and by using Definition 2 (iv), for $t>0$,

$$
\begin{aligned}
\frac{1}{\mathbb{M}_{r}\left(u, u^{*}, t\right)}-1= & \frac{1}{\mathbb{M}_{r}\left(\ell v, \ell v^{*}, t\right)}-1=\frac{1}{\mathbb{M}_{r}\left(A v, B v^{*}, t\right)}-1 \\
\leq & a\left(\frac{1}{\mathbb{M}_{r}\left(\ell v, \ell v^{*}, t\right)}-1\right) \\
& +b\left(\frac{\mathbb{M}_{r}\left(\ell v, \ell v^{*}, t\right)}{\mathbb{M}_{r}\left(\ell v^{*}, A v, 2 t\right) \cdot \mathbb{M}_{r}\left(\ell v, B v^{*}, 2 t\right)}-1\right) \\
& +c\left(\frac{1}{\mathbb{M}_{r}(\ell v, A v, t)}-1+\frac{1}{\mathbb{M}_{r}\left(\ell v^{*}, B v^{*}, t\right)}-1\right) \\
= & a\left(\frac{1}{\mathbb{M}_{r}\left(u, u^{*}, t\right)}-1\right) \\
& +b\left(\frac{\mathbb{M}_{r}\left(u, u^{*}, t\right)}{\mathbb{M}_{r}\left(u^{*}, u, 2 t\right) \cdot \mathbb{M}_{r}\left(u, u^{*}, 2 t\right)}-1\right) \\
& +c\left(\frac{1}{\mathbb{M}_{r}(u, u, t)}-1+\frac{1}{\mathbb{M}_{r}\left(u^{*}, u^{*}, t\right)}-1\right) \\
\leq & (a+b)\left(\frac{1}{\mathbb{M}_{r}\left(u, u^{*}, t\right)}-1\right),
\end{aligned}
$$

note that $(a+b)<1$, where $(a+b+2 c)<1$. Thus, we get that $\mathbb{M}_{r}\left(u, u^{*}, t\right)=1$, that is, $u=u^{*}$. By using the weak compatibility of the pair $(A, \ell),(B, \ell)$ and by using Preposition 9, we can get a unique CFP of the mappings $A, B$, and $\ell$. Let $\exists z \in W$ such that, $\ell z=A z=B z=z$. Hence, we get that $\mathbb{M}_{r}$ $(v, z, t)=1 \Rightarrow v=z$, for $t>0$.

Corollary 11. Let a fuzzy metric $\mathbb{M}_{r}$ is triangular in a complete FM space $\left(W, \mathbb{M}_{r}, *\right)$ and let $A, B, \ell: W \longrightarrow W$ be three self-mappings, satisfies for all $w, x \in W$,

$$
\begin{aligned}
& \frac{1}{\mathbb{M}_{r}(A w, B x, t)}-1 \leq a\left(\frac{1}{\mathbb{M}_{r}(\ell w, \ell x, t)}-1\right) \\
& +b\left(\frac{\mathbb{M}_{r}(\ell w, \ell x, t)}{\mathbb{M}_{r}(\ell x, A w, 2 t) \cdot \mathbb{M}_{r}(\ell w, B x, 2 t)}-1\right),
\end{aligned}
$$


for $t>0$ and $0 \leq a, b<1$ with $(a+b)<1$. If $A(W) \cup B(W)$ $\subset \ell(W)$, where $\ell(W)$ is a complete subspace of $W$. Then, $A, B$, and $\ell$ have a unique point of coincidence. Moreover, if the pairs $(A, \ell)$ and $(B, \ell)$ are weakly compatible. Then, $A, B$, and $\ell$ have a unique CFP in $W$.

If we use identity map instead of $\ell$, i.e., $\ell=I$, in Theorem 10 , we can get the following corollary:

Corollary 12. Let a fuzzy metric $\mathbb{M}_{r}$ is triangular in a complete FM space $\left(W, \mathbb{M}_{r}, *\right)$ and let $A, B: W \longrightarrow W$ be two self-mappings, satisfies for all $w, x \in W$,

$$
\begin{aligned}
\frac{1}{\mathbb{M}_{r}(A w, B x, t)}-1 \leq & a\left(\frac{1}{\mathbb{M}_{r}(w, x, t)}-1\right) \\
& +b\left(\frac{\mathbb{M}_{r}(w, x, t)}{\mathbb{M}_{r}(x, A w, 2 t) \cdot \mathbb{M}_{r}(w, B x, 2 t)}-1\right) \\
& +c\left(\frac{1}{\mathbb{M}_{r}(w, A w, t)}-1+\frac{1}{\mathbb{M}_{r}(x, B x, t)}-1\right)
\end{aligned}
$$

for $t>0,0 \leq a, b, c<1$ with $(a+b+2 c)<1$. Then, the mappings $A$ and $B$ have a unique CFP in $W$.

Remark 13. If we put the mappings $A=B$ and $\ell=I$ (identity map) with constant $c=0$ in Theorem 10, we obtained (Theorem 1 of [25]).
Example 14. Let $W=[0,1], *$ is a product continuous $t$ -norm on $W=[0,1]$ which is defined as $\xi * \zeta=\xi \cdot \zeta$ for all $\xi, \zeta \in W$ and a fuzzy metric $\mathbb{M}_{r}: W^{2} \times(0, \infty) \longrightarrow[0,1]$ is defined by

$$
\mathbb{M}_{r}(w, x, t)=\frac{t}{t+|w-x|}, \forall w, x \in W, \text { and } t>0
$$

Then, it is easy to prove that $\mathbb{M}_{r}$ is triangular and $(W$, $\left.\mathbb{M}_{r}, *\right)$ is a complete FM space. The mappings $A, B, \ell: W$ $\longrightarrow W$ be defined as

$$
A w=B w=\frac{4 w}{3 w+9} \text { and } \ell w=\frac{2 w}{3} \forall w \in W
$$

Then, from (29), we have

$$
\begin{aligned}
\frac{1}{\mathbb{M}_{r}(A w, B x, t)}-1 & =\frac{1}{t}|A w-B x|=\frac{1}{t}\left|\frac{4 w}{3 w+9}-\frac{4 x}{3 x+9}\right| \\
& =\left|\frac{36 w-36 x}{(3 w+9)(3 x+9)}\right| \leq\left|\frac{36 w-36 x}{81}\right| \\
& =\frac{2}{3}\left|\frac{\ell w-\ell x}{t}\right|=\frac{2}{3}\left(\frac{1}{\mathbb{M}_{r}(\ell w, \ell x, t)}-1\right) \text { for } t>0
\end{aligned}
$$

Hence, the self-mappings $A, B$, and $\ell$ are satisfied the weakly-compatible fuzzy-contraction condition in FM spaces. Next, we simplify the second term of (6), then, by using Definition 2 (iv) and from (29), for $t>0$, we have

$$
\begin{aligned}
& \frac{\mathbb{M}_{r}(\ell w, \ell x, t)}{\mathbb{M}_{r}(\ell x, A w, 2 t) \cdot \mathbb{M}_{r}(\ell w, B x, 2 t)}-1 \\
& \leq \frac{\mathbb{M}_{r}(\ell w, \ell x, t)}{\mathbb{M}_{r}(\ell x, \ell w, t) \cdot \mathbb{M}_{r}(\ell w, A w, t) \cdot \mathbb{M}_{r}(\ell w, \ell x, t) \cdot \mathbb{M}_{r}(\ell x, B x, t)}-1 \\
& =\frac{1}{\mathbb{M}_{r}(\ell x, \ell w, t) \cdot \mathbb{M}_{r}(\ell w, A w, t) \cdot \mathbb{M}_{r}(\ell x, B x, t)}-1 \\
& =\frac{(t+|\ell w-\ell x|) \cdot(t+|\ell w-A w|) \cdot(t+|\ell x-B x|)}{t^{3}}-1 \\
& =\frac{(t+|2 w / 3-2 x / 3|) \cdot(t+|2 w / 3-4 w / 3 w+9|) \cdot(t+|2 x / 3-4 w / 3 w+9|)}{t^{3}}-1 \\
& =\frac{(t+|2 w / 3-2 x / 3|) \cdot\left(t+\left|2\left(w^{2}+w\right) / 3 w+9\right|\right) \cdot\left(t+\left|2\left(x^{2}+x\right) / 3 x+9\right|\right)}{t^{3}}-1 \\
& =\frac{(t+|2 w / 3-2 x / 3|) \cdot\left(t^{2}+2 t\left(\left(w^{2}+w / 3 w+9\right)+\left(x^{2}+x / 3 x+9\right)\right)+\left(4\left(w^{2}+w\right) \cdot\left(x^{2}+x\right) /(3 w+9) \cdot(3 x+9)\right)\right)}{t^{3}}-1 \\
& \leq \frac{(t+2 / 3|w-x|) \cdot\left(t^{2}+(2 t / 81)\left(3 w^{2} x+3 w x^{2}+9\left(w^{2}+x^{2}\right)+6 w x+9(w+x)\right)+4 / 81\left(w^{2} x^{2}+w^{2} x+w x^{2}+w x\right)\right)}{t^{3}}-1 \\
= & \frac{(t+2 / 3|w-x|) \cdot\left(t^{2}+2 / 81\left(9 t\left(w^{2}+x^{2}\right)+2 w^{2} x^{2}+w x(6 t+2)+(w x(3 t+2)+9)(w+x)\right)\right)}{t^{3}}-1 \\
= & \frac{1}{t^{3}}\left(\frac{2 t^{2}}{3}|w-x|+\frac{2}{81}\left(t+\frac{2}{3}|w-x|\right)\left(9 t\left(w^{2}+x^{2}\right)+2 w^{2} x^{2}+w x(6 t+2)+(w x(3 t+2)+9)(w+x)\right)\right) .
\end{aligned}
$$


Lastly, we simplify the third term of (6), then from (29), for $t>0$,

$$
\begin{aligned}
& \frac{1}{\mathbb{M}_{r}(w, A w, t)}-1+\frac{1}{\mathbb{M}_{r}(x, B x, t)}-1 \\
& =\frac{1}{t}(|\ell w-A w|+|\ell x-B x|) \\
& \quad=\frac{1}{t}\left(\left|\frac{2 w}{3}-\frac{4 w}{3 w+9}\right|+\left|\frac{2 x}{3}-\frac{4 x}{3 x+9}\right|\right) \\
& =\frac{1}{t}\left(\frac{2\left(w^{2}+w\right)}{3 w+9}+\frac{2\left(x^{2}+x\right)}{3 x+9}\right) \\
& \leq \frac{2}{81 t}\left((3 w x+9)(w+x)+9\left(w^{2}+x^{2}\right)+6 w x\right) .
\end{aligned}
$$

Hence, all the conditions of Theorem 10 are satisfied with $a=2 / 3, b=1 / 9$, and $c=1 / 5$. The mappings $A, B$, and $\ell$ have a unique CFP, that is, 0 .

Theorem 15. Let a fuzzy metric $\mathbb{M}_{r}$ is triangular in a complete $F M$ space $\left(W, \mathbb{M}_{r}, *\right)$ and let $A, B, \ell: W \longrightarrow W$ be three self-mappings, satisfies for all $w, x \in W$,

$$
\begin{aligned}
& \frac{1}{\mathbb{M}_{r}(A w, B x, t)}-1 \leq a\left(\frac{1}{\mathbb{M}_{r}(\ell w, \ell x, t)}-1\right) \\
& \quad+b\left(\frac{1}{\mathbf{U}(A, B, \ell, w, x, t)}-1\right) \\
& \quad+c\left(\frac{\mathbb{M}_{r}(\ell w, A w, t) \cdot \mathbb{M}_{r}(\ell x, B x, t)}{\mathbb{M}_{r}(\ell w, B x, 2 t) \cdot \mathbb{M}_{r}(\ell w, \ell x, t) \cdot \mathbb{M}_{r}(\ell x, A w, 2 t)}-1\right),
\end{aligned}
$$

where

$\mathbf{U}(A, B, \ell, w, x, t)=\max \left\{\begin{array}{c}\mathbb{M}_{r}(\ell w, \ell x, t), \mathbb{M}_{r}(\ell w, A w, t), \\ \mathbb{M}_{r}(\ell x, B x, t), \mathbb{M}_{r}(\ell x, A w, t), \mathbb{M}_{r}(\ell w, B x, t)\end{array}\right\}$,

for $t>0$ and $0 \leq a, b, c<1$ with $(a+b+c)<1$. If $A(W) \cup B$ $(W) \subset \ell(W)$, where $\ell(W)$ is a complete subspace of $W$. Then $A, B$, and $\ell$ have a coincidence point in $W$.

Proof. Let $w_{0}$ be the arbitrary point of $W$. Using the condition $A(W) \cup B(W) \subset \ell(W)$ choose a sequence $\left(w_{i}\right)$ in $W$ such that

$$
\ell w_{2 i+1}=\mathrm{A} w_{2 i} \text { and } \ell w_{2 i+2}=B w_{2 i+1} \text {, for all } i \geq 0 \text {. }
$$

Now, by (34), for $t>0$,

$$
\begin{aligned}
& \frac{1}{\mathbb{M}_{r}\left(\ell w_{2 i+1}, \ell w_{2 i+2}, t\right)}-1=\frac{1}{\mathbb{M}_{r}\left(A w_{2 i}, B w_{2 i+1}, t\right)}-1 \\
& \leq a\left(\frac{1}{\mathbb{M}_{r}\left(\ell w_{2 i}, \ell w_{2 i+1}, t\right)}-1\right)+b\left(\frac{1}{\mathbf{U}\left(A, B, \ell, w_{2 i}, w_{2 i+1}, t\right)}-1\right) \\
& +c\left(\frac{\mathbb{M}_{r}\left(\ell w_{2 i}, A w_{2 i}, t\right) \cdot \mathbb{M}_{r}\left(\ell w_{2 i+1}, B w_{2 i+1}, t\right)}{\mathbb{M}_{r}\left(\ell w_{2 i}, B w_{2 i+1}, 2 t\right) \cdot \mathbb{M}_{r}\left(\ell w_{2 i}, \ell w_{2 i+1}, t\right) \cdot \mathbb{M}_{r}\left(\ell w_{2 i+1}, A w_{2 i}, 2 t\right)}-1\right) \\
& =a\left(\frac{1}{\mathbb{M}_{r}\left(\ell w_{2 i}, \ell w_{2 i+1}, t\right)}-1\right)+b\left(\frac{1}{\mathbf{U}\left(A, B, \ell, w_{2 i}, w_{2 i+1}, t\right)}-1\right) \\
& +c\left(\frac{\mathbb{M}_{r}\left(\ell w_{2 i}, \ell w_{2 i+1}, t\right) \cdot \mathbb{M}_{r}\left(\ell w_{2 i+1}, \ell w_{2 i+2}, t\right)}{\mathbb{M}_{r}\left(\ell w_{2 i}, \ell w_{2 i+2}, 2 t\right) \cdot \mathbb{M}_{r}\left(\ell w_{2 i}, \ell w_{2 i+1}, t\right)}-1\right),
\end{aligned}
$$

where

$$
\begin{aligned}
& \mathbf{U}\left(A, B, \ell, w_{2 i}, w_{2 i+1}, t\right) \\
& =\max \left\{\begin{array}{c}
\mathbb{M}_{r}\left(\ell w_{2 i}, \ell w_{2 i+1}, t\right), \mathbb{M}_{r}\left(\ell w_{2 i}, A w_{2 i}, t\right), \\
\mathbb{M}_{r}\left(\ell w_{2 i+1}, B w_{2 i+1}, t\right), \mathbb{M}_{r}\left(\ell w_{2 i+1}, A w_{2 i}, t\right), \mathbb{M}_{r}\left(\ell w_{2 i}, B w_{2 i+1}, t\right)
\end{array}\right\} \\
& \quad=\max \left\{\begin{array}{c}
\mathbb{M}_{r}\left(\ell w_{2 i}, \ell w_{2 i+1}, t\right), \mathbb{M}_{r}\left(\ell w_{2 i}, \ell w_{2 i+1}, t\right), \\
\mathbb{M}_{r}\left(\ell w_{2 i+1}, \ell w_{2 i+2}, t\right), \mathbb{M}_{r}\left(\ell w_{2 i+1}, \ell w_{2 i+1}, t\right), \mathbb{M}_{r}\left(\ell w_{2 i}, \ell w_{2 i+2}, t\right)
\end{array}\right\} \\
& =\max \left\{\mathbb{M}_{r}\left(\ell w_{2 i}, \ell w_{2 i+1}, t\right), \mathbb{M}_{r}\left(\ell w_{2 i+1}, \ell w_{2 i+2}, t\right), 1, \mathbb{M}_{r}\left(\ell w_{2 i}, \ell w_{2 i+2}, t\right)\right\}=1 .
\end{aligned}
$$

Now from (37), (38), and by using Definition 2 (iv), for $t>0$, we obtain

$$
\begin{aligned}
& \frac{1}{\mathbb{M}_{r}\left(\ell w_{2 i+1}, \ell w_{2 i+2}, t\right)}-1 \leq a\left(\frac{1}{\mathbb{M}_{r}\left(\ell w_{2 i}, \ell w_{2 i+1}, t\right)}-1\right) \\
& +c\left(\frac{\mathbb{M}_{r}\left(\ell w_{2 i}, \ell w_{2 i+1}, t\right) \cdot \mathbb{M}_{r}\left(\ell w_{2 i+1}, \ell w_{2 i+2}, t\right)}{\mathbb{M}_{r}\left(\ell w_{2 i}, \ell w_{2 i+1}, t\right) \cdot \mathbb{M}_{r}\left(\ell w_{2 i+1}, \ell w_{2 i+2}, t\right) \cdot \mathbb{M}_{r}\left(\ell w_{2 i}, \ell w_{2 i+1}, t\right)}-1\right)
\end{aligned}
$$

After simplification, for $t>0$,

$$
\begin{aligned}
\frac{1}{\mathbb{M}_{r}\left(\ell w_{2 i+1}, \ell w_{2 i+2}, t\right)}-1 & \leq \zeta\left(\frac{1}{\mathbb{M}_{r}\left(\ell w_{2 i}, \ell w_{2 i+1}, t\right)}-1\right) \text { where } \zeta \\
& =(a+c)<1
\end{aligned}
$$
$t>0$,

where $\zeta=(a+c)<1$. Similarly, again by view of (34), for

$$
\begin{aligned}
& \frac{1}{\mathbb{M}_{r}\left(\ell w_{2 i+2}, \ell w_{2 i+3}, t\right)}-1=\frac{1}{\mathbb{M}_{r}\left(A w_{2 i+2}, B w_{2 i+1}, t\right)}-1 \\
& \leq a\left(\frac{1}{\mathbb{M}_{r}\left(\ell w_{2 i+2}, \ell w_{2 i+1}, t\right)}-1\right)+b\left(\frac{1}{\mathbf{U}\left(A, B, \ell, w_{2 i+2}, w_{2 i+1}, t\right)}-1\right) \\
&+c\left(\frac{\mathbb{M}_{r}\left(\ell w_{2 i+2}, A w_{2 i+2}, t\right) \cdot \mathbb{M}_{r}\left(\ell w_{2 i+1}, B w_{2 i+1}, t\right)}{\mathbb{M}_{r}\left(\ell w_{2 i+2}, B w_{2 i+1}, 2 t\right) \cdot \mathbb{M}_{r}\left(\ell w_{2 i+2}, \ell w_{2 i+1}, t\right) \cdot \mathbb{M}_{r}\left(\ell w_{2 i+1}, A w_{2 i+2}, 2 t\right)}-1\right) \\
&= a\left(\frac{1}{\mathbb{M}_{r}\left(\ell w_{2 i+2}, \ell w_{2 i+1}, t\right)}-1\right)+b\left(\frac{1}{\mathbf{U}\left(A, B, \ell, w_{2 i+2}, w_{2 i+1}, t\right)}-1\right) \\
&+c\left(\frac{\mathbb{M}_{r}\left(\ell w_{2 i+2}, \ell w_{2 i+3}, t\right) \cdot \mathbb{M}_{r}\left(\ell w_{2 i+1}, \ell w_{2 i+2}, t\right)}{\mathbb{M}_{r}\left(\ell w_{2 i+2}, \ell w_{2 i+1}, t\right) \cdot \mathbb{M}_{r}\left(\ell w_{2 i+1}, \ell w_{2 i+3}, 2 t\right)}-1\right),
\end{aligned}
$$

where 


$$
\begin{aligned}
\mathbf{U} & \left(A, B, \ell, w_{2 i+2}, w_{2 i+1}, t\right) \\
& =\max \left\{\begin{array}{c}
\mathbb{M}_{r}\left(\ell w_{2 i+2}, \ell w_{2 i+1}, t\right), \mathbb{M}_{r}\left(\ell w_{2 i+2}, A w_{2 i+2}, t\right), \\
\mathbb{M}_{r}\left(\ell w_{2 i+1}, B w_{2 i+1}, t\right), \mathbb{M}_{r}\left(\ell w_{2 i+1}, A w_{2 i+2}, t\right), \mathbb{M}_{r}\left(\ell w_{2 i+2}, B w_{2 i+1}, t\right)
\end{array}\right\} \\
& =\max \left\{\begin{array}{c}
\mathbb{M}_{r}\left(\ell w_{2 i+2}, \ell w_{2 i+1}, t\right), \mathbb{M}_{r}\left(\ell w_{2 i+2}, \ell w_{2 i+3}, t\right), \\
\mathbb{M}_{r}\left(\ell w_{2 i+1}, \ell w_{2 i+2}, t\right), \mathbb{M}_{r}\left(\ell w_{2 i+2}, \ell w_{2 i+3}, t\right), \mathbb{M}_{r}\left(\ell w_{2 i+2}, \ell w_{2 i+2}, t\right)
\end{array}\right\} \\
& =\max \left\{\mathbb{M}_{r}\left(\ell w_{2 i+2}, \ell w_{2 i+1}, t\right), \mathbb{M}_{r}\left(\ell w_{2 i+2}, \ell w_{2 i+3}, t\right), \mathbb{M}_{r}\left(\ell w_{2 i+1}, \ell w_{2 i+3}, t\right), 1\right\} \\
& =1 .
\end{aligned}
$$

Now from (41), (42), and by using Definition 2 (iv),

$$
\begin{aligned}
& \frac{1}{\mathbb{M}_{r}\left(\ell w_{2 i+2}, \ell w_{2 i+3}, t\right)}-1 \leq a\left(\frac{1}{\mathbb{M}_{r}\left(\ell w_{2 i+2}, \ell w_{2 i+1}, t\right)}-1\right) \\
& \quad+c\left(\frac{\mathbb{M}_{r}\left(\ell w_{2 i+2}, \ell w_{2 i+3}, t\right) \cdot \mathbb{M}_{r}\left(\ell w_{2 i+1}, \ell w_{2 i+2}, t\right)}{\mathbb{M}_{r}\left(\ell w_{2 i+2}, \ell w_{2 i+1}, t\right) \cdot \mathbb{M}_{r}\left(\ell w_{2 i+1}, \ell w_{2 i+2}, t\right) \cdot \mathbb{M}_{r}\left(\ell w_{2 i+2}, \ell w_{2 i+3}, t\right)}-1\right) .
\end{aligned}
$$

Then after simplification, for $t>0$,

$$
\begin{aligned}
\frac{1}{\mathbb{M}_{r}\left(\ell w_{2 i+2}, \ell w_{2 i+3}, t\right)}-1 & \leq \zeta\left(\frac{1}{\mathbb{M}_{r}\left(\ell w_{2 i+1}, \ell w_{2 i+2}, t\right)}-1\right) \text { where } \zeta \\
& =(a+c)<1
\end{aligned}
$$

Now, from (40), (44), and by induction,

$$
\begin{aligned}
\frac{1}{\mathbb{M}_{r}\left(\ell w_{2 i+2}, \ell w_{2 i+3}, t\right)}-1 & \leq \zeta\left(\frac{1}{\mathbb{M}_{r}\left(\ell w_{2 i+1}, \ell w_{2 i+2}, t\right)}-1\right) \\
& \leq \zeta^{2}\left(\frac{1}{\mathbb{M}_{r}\left(\ell w_{2 i}, \ell w_{2 i+1}, t\right)}-1\right) \\
& \leq \cdots \leq \zeta^{2 i+2}\left(\frac{1}{\mathbb{M}_{r}\left(\ell w_{0}, \ell w_{1}, t\right)}-1\right) \\
& \longrightarrow 0 \text { as } i \longrightarrow \infty .
\end{aligned}
$$

Hence, $\left(\ell w_{i}\right)$ is a fuzzy-contractive sequence in $\left(W, \mathbb{M}_{r}\right.$, $*)$, therefore

$$
\lim _{i \longrightarrow \infty} \mathbb{M}_{r}\left(\ell w_{i}, \ell w_{i-1}, t\right)=1 \text { for } t>0 .
$$

Since $\mathbb{M}_{r}$ is triangular, $j>i>i_{0}$,

$$
\begin{aligned}
\frac{1}{\mathbb{M}_{r}\left(\ell w_{i}, \ell w_{j}, t\right)}-1 \leq & \left(\frac{1}{\mathbb{M}_{r}\left(\ell w_{i}, \ell w_{i+1}, t\right)}-1\right)+\left(\frac{1}{\mathbb{M}_{r}\left(\ell w_{i+1}, \ell w_{i+2}, t\right)}-1\right) \\
& +\cdots+\left(\frac{1}{\mathbb{M}_{r}\left(\ell w_{i-1}, \ell w_{j}, t\right)}-1\right) \\
\leq & \zeta^{i}\left(\frac{1}{\mathbb{M}_{r}\left(\ell w_{i}, \ell w_{i+1}, t\right)}-1\right)+\zeta^{i+1}\left(\frac{1}{\mathbb{M}_{r}\left(\ell w_{i+1}, \ell w_{i+2}, t\right)}-1\right) \\
& +\cdots+\zeta^{j-1}\left(\frac{1}{\mathbb{M}_{r}\left(\ell w_{i-1}, \ell w_{j}, t\right)}-1\right) \\
\leq & \left(\zeta^{i}+\zeta^{i+1}+\cdots+\zeta^{j-1}\right)\left(\frac{1}{\mathbb{M}_{r}\left(\ell w_{0}, \ell w_{1}, t\right)}-1\right) \\
\leq & \left(\frac{\zeta^{i}}{1-\zeta}\right)\left(\frac{1}{\mathbb{M}_{r}\left(\ell w_{0}, \ell w_{1}, t\right)}-1\right) \longrightarrow 0 \text { as } i \longrightarrow \infty .
\end{aligned}
$$

This shows that $\left(\ell w_{i}\right)$ is a Cauchy sequence and $\ell(W)$ is a complete subspace of $W$. Hence, $\exists u, v \in W$ such that $\ell w_{i}$ $\longrightarrow u=\ell v$ as $i \longrightarrow \infty$, i.e.,

$$
\lim _{i \longrightarrow \infty} \mathbb{M}_{r}\left(u, \ell w_{i}, t\right)=\mathbb{M}_{r}(u, \ell v, t)=1 \text { for } t>0 .
$$

Since $\mathbb{M}_{r}$ is triangular,

$$
\begin{aligned}
\frac{1}{\mathbb{M}_{r}(\ell v, A v, t)}-1 \leq & \left(\frac{1}{\mathbb{M}_{r}\left(\ell v, \ell w_{2 i+2}, t\right)}-1\right) \\
& +\left(\frac{1}{\mathbb{M}_{r}\left(\ell w_{2 i+2}, A v, t\right)}-1\right) \text { for } t>0 .
\end{aligned}
$$

Now, from (34), (46), (48), and by using Definition 2 (iv), for $t>0$, we have that

$$
\begin{aligned}
& \frac{1}{\mathbb{M}_{r}\left(\ell w_{2 i+2}, A v, t\right)}-1=\left(\frac{1}{\mathbb{M}_{r}\left(A v, B w_{2 i+1}, t\right)}-1\right) \\
& \leq a\left(\frac{1}{\mathbb{M}_{r}\left(\ell v, \ell w_{2 i+1}, t\right)}-1\right)+b\left(\frac{1}{\mathbf{U}\left(A, B, \ell, v, w_{2 i+1}, t\right)}-1\right) \\
& \quad+c\left(\frac{\mathbb{M}_{r}(\ell v, A v, t) \cdot \mathbb{M}_{r}\left(\ell w_{2 i+1}, B w_{2 i+1}, t\right)}{\mathbb{M}_{r}\left(\ell v, B w_{2 i+1}, 2 t\right) \cdot \mathbb{M}_{r}\left(\ell v, \ell w_{2 i+1}, t\right) \cdot \mathbb{M}_{r}\left(\ell w_{2 i+1}, A v, 2 t\right)}-1\right) \\
& \leq a\left(\frac{1}{\mathbb{M}_{r}\left(\ell v, \ell w_{2 i+1}, t\right)}-1\right)+b\left(\frac{1}{\mathbf{U}\left(A, B, \ell, v, w_{2 i+1}, t\right)}-1\right) \\
& \quad+c\left(\frac{\mathbb{M}_{r}(\ell v, A v, t) \cdot \mathbb{M}_{r}\left(\ell w_{2 i+1}, \ell w_{2 i+2}, t\right)}{\mathbb{M}_{r}\left(\ell v, \ell w_{2 i+2}, 2 t\right) \cdot \mathbb{M}_{r}\left(\ell v, \ell w_{2 i+1}, t\right) \cdot \mathbb{M}_{r}\left(\ell w_{2 i+1}, \ell v, t\right) \cdot \mathbb{M}_{r}(\ell v, A v, t)}-1\right) \\
& \longrightarrow b\left(\frac{1}{\mathbf{U}\left(A, B, \ell, \mathrm{v}, w_{2 i+1}, t\right)}-1\right) \text { as } i \longrightarrow \infty,
\end{aligned}
$$

where

$$
\begin{aligned}
& \mathbf{U}\left(A, B, \ell, v, w_{2 i+1}, t\right) \\
& =\max \left\{\begin{array}{c}
\mathbb{M}_{r}\left(\ell v, \ell w_{2 i+1}, t\right), \mathbb{M}_{r}(\ell v, A v, t), \\
\mathbb{M}_{r}\left(\ell w_{2 i+1}, B w_{2 i+1}, t\right), \mathbb{M}_{r}\left(\ell w_{2 i+1}, A v, t\right), \mathbb{M}_{r}\left(\ell v, B w_{2 i+1}, t\right)
\end{array}\right\} \\
& \mathbb{M}_{r}\left(\ell v, \ell w_{2 i+1}, t\right), \mathbb{M}_{r}(\ell v, A v, t), \\
& =\max \left\{\begin{array}{c}
\longrightarrow \\
\mathbb{M}_{r}\left(\ell w_{2 i+1}, \ell w_{2 i+2}, t\right), \mathbb{M}_{r}\left(\ell w_{2 i+1}, A v, t\right), \mathbb{M}_{r}\left(\ell v, \ell w_{2 i+2}, t\right)
\end{array}\right\} \\
& \longrightarrow \max \left\{1, \mathbb{M}_{r}(\ell v, A v, t)\right\}=1 \text { as } i \longrightarrow \infty .
\end{aligned}
$$

Now from (50) and (51), for $t>0$, we have

$$
\lim _{i \longrightarrow \infty} \sup \left(\frac{1}{\mathbb{M}_{r}\left(\ell w_{2 i+2}, A v, t\right)}-1\right)=0 \text { for } t>0 \text {. }
$$

By using the value (48) and (52) in (49) with limit $i$ $\longrightarrow \infty$, we get that $\mathbb{M}_{r}(\ell v, A v, t)=1 \Rightarrow u=\ell v=A v$ for $t>$ 0 . Next, we have to prove that $u=\ell v=B v$. Since, $\mathbb{M}_{r}$ is triangular,

$$
\begin{aligned}
\frac{1}{\mathbb{M}_{r}(\ell v, B v, t)}-1 \leq & \left(\frac{1}{\mathbb{M}_{r}\left(\ell v, \ell w_{2 i+1}, t\right)}-1\right) \\
& +\left(\frac{1}{\mathbb{M}_{r}\left(\ell w_{2 i+1}, B v, t\right)}-1\right) \text { for } t>0 .
\end{aligned}
$$


Now, from (34), (46), (48), and by using Definition 2 (iv), for $t>0$, we have that

$$
\begin{aligned}
& \frac{1}{\mathbb{M}_{r}\left(\ell w_{2 i+1}, B v, t\right)}-1=\left(\frac{1}{\mathbb{M}_{r}\left(A w_{2 i}, B v, t\right)}-1\right) \\
& \leq a\left(\frac{1}{\mathbb{M}_{r}\left(\ell w_{2 i}, \ell v, t\right)}-1\right)+b\left(\frac{1}{\mathbf{U}\left(A, B, \ell, w_{2 i}, v, t\right)}-1\right) \\
& \quad+c\left(\frac{\mathbb{M}_{r}\left(\ell w_{2 i}, A w_{2 i}, t\right) \cdot \mathbb{M}_{r}(\ell v, B v, t)}{\mathbb{M}_{r}\left(\ell w_{2 i}, B v, 2 t\right) \cdot \mathbb{M}_{r}\left(\ell w_{2 i}, \ell w_{2 i}, t\right) \cdot \mathbb{M}_{r}\left(\ell v, A w_{2 i}, 2 t\right)}-1\right) \\
& \leq a\left(\frac{1}{\mathbb{M}_{r}\left(\ell w_{2 i}, \ell v, t\right)}-1\right)+b\left(\frac{1}{\mathbf{U}\left(A, B, \ell, w_{2 i}, v, t\right)}-1\right) \\
& \quad c\left(\frac{\mathbb{M}_{r}\left(\ell w_{2 i}, \ell w_{2 i+1}, t\right) \cdot \mathbb{M}_{r}(\ell v, B v, t)}{\mathbb{M}_{r}\left(\ell w_{2 i}, \ell v, t\right) \cdot \mathbb{M}_{r}(\ell v, B v, t) \cdot \mathbb{M}_{r}\left(\ell w_{2 i}, \ell v, t\right) \cdot \mathbb{M}_{r}\left(\ell v, \ell w_{2 i+1}, t\right)}-1\right) \\
& \longrightarrow b\left(\frac{1}{\mathbf{U}\left(A, B, \ell, v, w_{2 i}, t\right)}-1\right) \text { as } i \longrightarrow \infty
\end{aligned}
$$

where

$$
\begin{aligned}
& \mathbf{U}\left(A, B, \ell, w_{2 i}, v, t\right) \\
& =\max \left\{\begin{array}{c}
\mathbb{M}_{r}\left(\ell w_{2 i}, \ell v, t\right), \mathbb{M}_{r}\left(\ell w_{2 i}, A w_{2 i}, t\right), \\
\mathbb{M}_{r}(\ell v, B v, t), \mathbb{M}_{r}\left(\ell v, A w_{2 i}, t\right), \mathbb{M}_{r}\left(\ell w_{2 i}, B v, t\right)
\end{array}\right\} \\
& \quad=\max \left\{\begin{array}{c}
\mathbb{M}_{r}\left(\ell w_{2 i}, \ell v, t\right), \mathbb{M}_{r}\left(\ell w_{2 i}, \ell w_{2 i+1}, t\right), \\
\mathbb{M}_{r}(\ell v, B v, t), \mathbb{M}_{r}\left(\ell v, \ell w_{2 i+1}, t\right), \mathbb{M}_{r}\left(\ell w_{2 i}, B v, t\right)
\end{array}\right\} \\
& \longrightarrow \max \left\{1, \mathbb{M}_{r}(\ell v, B v, t)\right\}=1 \text { as } i \longrightarrow \infty .
\end{aligned}
$$

Now from (54) and (55), for $t>0$,

$$
\lim _{i \longrightarrow \infty} \sup \left(\frac{1}{\mathbb{M}_{r}\left(\ell w_{2 i+1}, B v, t\right)}-1\right)=0 \text { for } t>0 .
$$

By using the value (48) and (56) in (53) with limit $i$ $\longrightarrow \infty$, we obtain $\mathbb{M}_{r}(\ell v, B v, t)=1 \Rightarrow u=\ell v=B v$ for $t>0$. Hence, we obtain that $u$ is a common coincidence point of the mappings $\ell, A$, and $B$ in $W$ such that $u=\ell v=A v=B v$.

Corollary 16. Let a fuzzy metric $\mathbb{M}_{r}$ is triangular in a complete FM space $\left(W, \mathbb{M}_{r}, *\right)$ and let $A, B, \ell: W \longrightarrow W$ be three self-mappings, satisfies for all $w, x \in W$,

$\frac{1}{\mathbb{M}_{r}(A w, B x, t)}-1 \leq a\left(\frac{1}{\mathbb{M}_{r}(\ell w, \ell x, t)}-1\right)+b\left(\frac{1}{\mathbf{U}(A, B, \ell, w, x, t)}-1\right)$,

where

$\mathbf{U}(A, B, \ell, w, x, t)=\max \left\{\begin{array}{c}\mathbb{M}_{r}(\ell w, \ell x, t), \mathbb{M}_{r}(\ell w, A w, t), \\ \mathbb{M}_{r}(\ell x, B x, t), \mathbb{M}_{r}(\ell x, A w, t), \mathbb{M}_{r}(\ell w, B x, t)\end{array}\right\}$,

for $t>0$ and $0 \leq a, b<1$ with $(a+b)<1$. If $A(W) \cup B(W)$ $\subset \ell(W)$, where $\ell(W)$ is a complete subspace of $W$. Then $A$, $B$, and $\ell$ have a coincidence point in $W$. Moreover, if the pairs $(A, \ell)$ and $(B, \ell)$ are weakly compatible. Then, $A, B$, and $\ell$ have a unique CFP in $W$.
Proof. From the proof of Theorem 15, $u$ is a common coincidence point of the mappings $\ell, A$, and $B$ in $W$ such that $u$ $=\ell v=A v=B v$ for some $v \in W$. Now we prove the uniqueness of the coincidence point, let $\exists u^{*} \in W$ is another common coincidence point of $\ell, A$, and $B$ in $W$ such that $u^{*}=\ell v^{*}=A v^{*}=B v^{*}$ for some $v^{*} \in W$. Then from (57), for $t>0$,

$$
\begin{aligned}
\frac{1}{\mathbb{M}_{r}\left(u, u^{*}, t\right)}-1= & \frac{1}{\mathbb{M}_{r}\left(\ell v, \ell v^{*}, t\right)}-1=\frac{1}{\mathbb{M}_{r}\left(A v, B v^{*}, t\right)}-1 \\
\leq & a\left(\frac{1}{\mathbb{M}_{r}\left(\ell v, \ell v^{*}, t\right)}-1\right) \\
& +b\left(\frac{1}{\mathbf{U}\left(A, B, \ell, v, v^{*}, t\right)}-1\right),
\end{aligned}
$$

where

$$
\begin{aligned}
& \mathbf{U}\left(A, B, \ell, v, v^{*}, t\right) \\
& \quad=\max \left\{\begin{array}{c}
\mathbb{M}_{r}\left(\ell v, \ell v^{*}, t\right), \mathbb{M}_{r}(\ell v, A v, t), \\
\mathbb{M}_{r}\left(\ell v^{*}, B v^{*}, t\right), \mathbb{M}_{r}\left(\ell v^{*}, A v, t\right), \mathbb{M}_{r}\left(\ell v, B v^{*}, t\right)
\end{array}\right\} \\
& \quad=\max \left\{\mathbb{M}_{r}\left(\ell v, \ell v^{*}, t\right), 1\right\}=1 .
\end{aligned}
$$

Thus,

$$
\begin{aligned}
\frac{1}{\mathbb{M}_{r}\left(u, u^{*}, t\right)}-1 & \leq a\left(\frac{1}{\mathbb{M}_{r}\left(\ell v, \ell v^{*}, t\right)}-1\right) \\
& =a\left(\frac{1}{\mathbb{M}_{r}\left(u, u^{*}, t\right)}-1\right) \text { for } t>0 .
\end{aligned}
$$

Noticing that $(1-a) \neq 0$ where $(a+b)<1$, therefore, $\mathbb{M}_{r}\left(u, u^{*}, t\right)=1 \Rightarrow u=u^{*}$ for $t>0$. By using the weak compatibility of the pair $(A, \ell),(B, \ell)$ and by Preposition 9 , we can get a unique CFP of the mappings $A, B$, and $\ell$. Let $\exists z$ $\in W$ such that, $\ell z=A z=B z=z$. Hence, we get that $\mathbb{M}_{r}(v, z$ $, t)=1 \Rightarrow v=z$, for $t>0$.

Example 17. From Example 14, it is proved that the three self-mappings $A, B$, and $\ell$ are weakly compatible fuzzycontractive in FM-spaces, that is,

$$
\frac{1}{\mathbb{M}_{r}(A w, \mathrm{~B} x, t)}-1=\frac{2}{3}\left(\frac{1}{\mathbb{M}_{r}(\ell w, \ell x, t)}-1\right) \text { for } t>0 .
$$

Next, we calculate the value of the second term, present in (57). Then, we have the following cases: 
(1) If the maximum value of $\mathbf{U}(A, B, \ell, w, x, t)=\mathbb{M}_{r}(\ell w$ $, \ell x, t)$, for $t>0$. Then, by using (29)

$$
\begin{aligned}
\frac{1}{\mathbf{U}(A, B, \ell, w, x, t)}-1 & =\frac{1}{\mathbb{M}_{r}(\ell w, \ell x, t)}-1=\frac{1}{t}|\ell w-\ell x| \\
& =\frac{2}{3 t}|w-x| \text { for } t>0 .
\end{aligned}
$$

(2) If the maximum value of $\mathbf{U}(A, B, \ell, w, x, t)=\mathbb{M}_{r}(\ell w$ , $A w, t)$, for $t>0$. Then, by using (29)

$$
\begin{aligned}
& \frac{1}{\mathbf{U}(A, B, \ell, w, x, t)}-1=\frac{1}{\mathbb{M}_{r}(\ell w, A w, t)}-1=\frac{1}{t}|\ell w-A w| \\
& =\frac{1}{t}\left|\frac{2 w}{3}-\frac{4 w}{3 w+9}\right|=\frac{1}{t}\left|\frac{2\left(w^{2}+w\right)}{3 w+9}\right| \leq \frac{2}{9 t}\left(w^{2}+w\right) \text { for } t>0 .
\end{aligned}
$$

(3) If the maximum value of $\mathbf{U}(A, B, \ell, w, x, t)=\mathbb{M}_{r}(\ell x$, $B x, t)$, for $t>0$. Then, by using (29)

$$
\begin{aligned}
& \frac{1}{\mathbf{U}(A, B, \ell, w, x, t)}-1=\frac{1}{\mathbb{M}_{r}(\ell x, B x, t)}-1=\frac{1}{t}|\ell x-B x| \\
& =\frac{1}{t}\left|\frac{2 x}{3}-\frac{4 x}{3 x+9}\right|=\frac{1}{t}\left|\frac{2\left(x^{2}+x\right)}{3 x+9}\right| \leq \frac{2}{9 t}\left(x^{2}+x\right) \text { for } t>0 .
\end{aligned}
$$

(4) If the maximum value of $\mathbf{U}(A, B, \ell, w, x, t)=\mathbb{M}_{r}(\ell x$, $A w, t)$, for $t>0$. Then, by using (29)

$$
\begin{aligned}
& \frac{1}{\mathrm{U}(A, B, \ell, w, x, t)}-1=\frac{1}{\mathbb{M}_{r}(\ell x, A w, t)}-1=\frac{1}{t}|\ell x-A w| \\
& \quad=\frac{1}{t}\left|\frac{2 x}{3}-\frac{4 w}{3 w+9}\right|=\frac{1}{t}\left|\frac{2(w x+3 x-2 w)}{3 x+9}\right| \\
& \leq \frac{2}{9 t}|w x+3 x-2 w| \text { for } t>0 .
\end{aligned}
$$

(5) If the maximum value of $\mathbf{U}(A, B, \ell, w, x, t)=\mathbb{M}_{r}(\ell w$ $, B x, t)$, for $t>0$. Then, by using (29)

$$
\begin{aligned}
& \frac{1}{\mathbf{U}(A, B, \ell, w, x, t)}-1=\frac{1}{\mathbb{M}_{r}(\ell w, B x, t)}-1=\frac{1}{t}|\ell w-B x| \\
& \quad=\frac{1}{t}\left|\frac{2 w}{3}-\frac{4 x}{3 x+9}\right|=\frac{1}{t}\left|\frac{2(w x+3 w-2 x)}{3 w+9}\right| \\
& \quad \leq \frac{2}{9 t}|w x+3 w-2 x| \text { for } t>0 .
\end{aligned}
$$

Hence, all the cases together with (62) and with contacts $a=2 / 3$ and $b=2 / 7$, we have

$$
\begin{aligned}
\frac{1}{\mathbb{M}_{r}(A w, B x, t)}-1 & \leq \frac{2}{3}\left(\frac{1}{\mathbb{M}_{r}(\ell w, \ell x, t)}-1\right) \\
& +\frac{2}{7}\left(\frac{1}{\mathbf{U}(A, B, \ell, w, x, t)}-1\right),
\end{aligned}
$$

where

$$
\begin{aligned}
& \mathbf{U}(A, B, \ell, w, x, t) \\
& \quad=\max \left\{\begin{array}{c}
\mathbb{M}_{r}(\ell w, \ell x, t), \mathbb{M}_{r}(\ell w, A w, t), \\
\mathbb{M}_{r}(\ell x, B x, t), \mathbb{M}_{r}(\ell x, A w, t), \mathbb{M}_{r}(\ell w, B x, t)
\end{array}\right\} \text { for } t>0 .
\end{aligned}
$$

Thus, all the hypotheses of Corollary 16 are satisfied with $a=2 / 3, b=2 / 7$, and the mappings $A, B$, and $\ell$ have a unique CFP, namly, 0 .

\section{Application to the Fuzzy Differential Equations}

In this section, we present an application of the fuzzy differential equations (FDEs) to support our main work. From the book of Lakshmikantham and Mohapatra [32], we have the following FDEs.

Let $\mathbf{F}$ be the space of all fuzzy subsets $w$ of $\mathscr{R}$ where $w: \mathscr{R} \longrightarrow[0,1]$.

$$
\begin{aligned}
& w^{\prime \prime}(v)=h\left(v, w(v), w^{\prime}(v)\right), v \in \mathcal{J}=[a, b], \\
& w\left(v_{1}\right)=w_{1}, w\left(v_{2}\right)=w_{2}, v_{1}, v_{2} \in \mathcal{J}=[a, b],
\end{aligned}
$$

where $h: \mathscr{J} \times \mathbf{F} \times \mathbf{F} \longrightarrow \mathbf{F}$ is a continuous function. This problem is equivalent to the integral equation

$$
w(v)=\int_{v_{1}}^{v_{2}} Q(v, \xi)\left(h\left(\xi, w(\xi), w^{\prime}(\xi)\right)\right) d \xi+\mathscr{B}(v),
$$

where Green's function $Q$ is given by

$$
Q(v, \xi)= \begin{cases}\frac{\left(v_{2}-v\right)\left(\xi-v_{1}\right)}{v_{2}-v_{1}}, & v_{1} \leq \xi \leq v \leq v_{2}, \\ \frac{\left(v_{2}-\xi\right)\left(v-v_{1}\right)}{v_{2}-v_{1}}, & v_{1} \leq v \leq \xi \leq v_{2} .\end{cases}
$$

And $\mathscr{B}(v)$ satisfies $\mathscr{B}^{\prime \prime}=0, \mathscr{B}\left(v_{1}\right)=w_{1}, \mathscr{B}\left(v_{2}\right)=w_{2}$. Here, we recall some properties of $Q(v, \xi)$, that is,

$$
\begin{aligned}
& \int_{v_{1}}^{v_{2}} Q(v, \xi) d \xi \leq \frac{\left(v_{2}-v_{1}\right)^{2}}{8}, \\
& \int_{v_{1}}^{v_{2}} Q_{v}(v, \xi) d \xi \leq \frac{v_{2}-v_{1}}{2} .
\end{aligned}
$$

Let $C=C^{1}(\mathscr{J}, \mathbf{F}), *$ is a continuous $t$-norm, and a fuzzy metric $\mathbb{M}_{r}: C \times C \times(0, \infty) \longrightarrow[0,1]$ be defined as 


$$
\mathbb{M}_{r}(w, x, t)=\frac{t}{t+\mathfrak{D}(w, x)} \text { where } \mathfrak{D}(w, x)=|w-x|,
$$

$\forall w, x \in C$, and $t>0$. Then, it is easy to prove that $\mathbb{M}_{r}$ is triangular and $\left(C, \mathbb{M}_{r}, *\right)$ is a complete FM-space.

Now, we prove the existing result for the above boundary value problem by using Corollary 16 .

Theorem 18. Assume that $h_{1}, h_{2}: \mathcal{J} \times F \times F \longrightarrow F$ and let there exist $\alpha, \beta \in(0,1)$ with $\alpha \leq \beta$ such that for all $w, x \in C^{1}$ $(\mathscr{F}, F)$, satisfies

$$
\begin{gathered}
\left|h_{1}\left(v, w(v), w^{\prime}(v)\right)-h_{2}\left(v, x(v), x^{\prime}(v)\right)\right| \\
\quad \leq \alpha|w(v)-x(v)|+\beta\left|w^{\prime}(v)-x^{\prime}(v)\right| .
\end{gathered}
$$

Let there exists $\eta \in(0,1)$ such that

$$
\mathfrak{D}(w(v), x(v))=\mathfrak{D}(w, x) \leq \eta \mathbf{M}(A, B, \ell, w, x),
$$

where

$$
\begin{aligned}
\mathbf{M}(A, B, \ell, w, x)= & \max \{|\ell w-\ell x|,|\ell w-A w|, \\
& \cdot|\ell x-B x|,|\ell x-A w|,|\ell w-B x|\} .
\end{aligned}
$$

Then the integral equations

$$
\begin{gathered}
w(v)=\int_{v_{1}}^{v_{2}} Q(v, \xi)\left(h_{1}\left(\xi, w(\xi), w^{\prime}(\xi)\right) d \xi+\mathscr{B}(v), v \in \mathscr{J},\right. \\
x(v)=\int_{v_{1}}^{v_{2}} Q(v, \xi)\left(h_{2}\left(\xi, x(\xi), x^{\prime}(\xi)\right) d \xi+\mathscr{B}(v), v \in \mathscr{J},\right.
\end{gathered}
$$

have a unique common solution in $C^{1}\left[\left[v_{1}, v_{2}\right], \mathbf{F}\right]$.

Proof. Suppose that $C=\left[\left[v_{1}, v_{2}\right], \mathrm{F}\right]$ with metric

$$
\mathfrak{D}(w, x)=\max _{v_{1} \leq v \leq v_{2}}\left(\alpha|w(v)-x(v)|+\beta\left|w^{\prime}(v)-x^{\prime}(v)\right|\right) .
$$

The space $(C, \mathfrak{D})$ is a complete metric space. Now, we define the operators $A, B, \ell: C \longrightarrow C$ as

$$
A(w)=G_{w}+\mathscr{B}, B(x)=H_{x}+\mathscr{B}, \ell(w)=w \text {, and } \ell(x)=x,
$$

where

$$
\begin{aligned}
& G_{w}(v)=\int_{v_{1}}^{v_{2}} Q(v, \xi)\left(h_{1}\left(\xi, w(\xi), w^{\prime}(\xi)\right) d \xi, v \in \mathcal{F},\right. \\
& H_{x}(v)=\int_{v_{1}}^{v_{2}} Q(v, \xi)\left(h_{2}\left(\xi, x(\xi), x^{\prime}(\xi)\right) d \xi, v \in \mathcal{F},\right.
\end{aligned}
$$

where $h_{1}, h_{2} \in C(\mathscr{J} \times \mathbf{F} \times \mathbf{F}, \mathbf{F}), w, x \in C^{1}(\mathscr{F}, \mathbf{F})$, and $\mathscr{B} \in C($ $\mathscr{J}, \mathbf{F})$. Now by the properties of $Q(v, \xi)$, and from (79), (80) and by using the hypothesis, we have

$$
\begin{aligned}
&|A w(v)-B x(v)| \leq \int_{v_{1}}^{v_{2}}|Q(v, \xi)|\left|h_{1}\left(\xi, w(\xi), w^{\prime}(\xi)\right)-h_{2}\left(\xi, x(\xi), x^{\prime}(\xi)\right)\right| d \xi \\
& \leq \mathfrak{D}(w, x) \int_{v_{1}}^{v_{2}}|Q(v, \xi)| d \xi \leq \frac{\left(v_{2}-v_{1}\right)^{2}}{8} \mathfrak{D}(w, x) \leq \frac{\mathfrak{D}(w, x)}{8}, \\
&\left|(A w)^{\prime}(v)-(B x)^{\prime}(v)\right| \leq \int_{v_{1}}^{v_{2}}\left|Q_{v}(v, \xi)\right|\left|h_{1}\left(\xi, w(\xi), w^{\prime}(\xi)\right)-h_{2}\left(\xi, x(\xi), x^{\prime}(\xi)\right)\right| d \xi \\
& \leq \mathfrak{D}(w, x) \int_{v_{1}}^{v_{2}}\left|Q_{v}(v, \xi)\right| d \xi \leq \frac{v_{2}-v_{1}}{2} \mathfrak{D}(w, x) \leq \frac{\mathfrak{D}(w, x)}{2} .
\end{aligned}
$$

Now, from the above and by view of (75), and (79), we have that

$$
\begin{gathered}
\mathfrak{D}(A w, B x)(83)=\max _{v_{1} \leq v \leq v_{2}}\left(\alpha|A w(v)-B x(v)|+\beta\left|(A w)^{\prime}(v)-(B x)^{\prime}(v)\right|\right) \\
\leq \alpha \frac{\mathfrak{D}(w, x)}{8}+\beta \frac{\mathfrak{D}(w, x)}{2} \leq\left(\frac{5}{8} \beta\right) \mathfrak{D}(w, x) .
\end{gathered}
$$

Now, from (76), we have that

$$
\mathfrak{D}(A w, B x) \leq\left(\frac{5}{8} \beta\right) \mathfrak{D}(w, x) \leq \lambda \mathbf{M}(A, B, \ell, w, x),
$$

where $\lambda=5 / 8 \beta \eta<1$. Now we are in the position to apply Corollary 16 to get that the mappings $A, B$, and $\ell$ have a unique CFP $w^{*} \in C$, i.e., $w^{*}$ is a solution of the BVP. We have the following cases.

(1) If $|\ell w-\ell x|$ is the maximum term in (77), then $\mathbf{M}($ $A, B, \ell, w, x)=|\ell w-\ell x|$. Now from (74) and (84), we have

$$
\begin{aligned}
\frac{1}{\mathbb{M}_{r}(A w, B x, t)}-1 & =\frac{\mathfrak{D}(A w, B x)}{t} \leq \lambda \frac{\mathbf{M}(A, B, \ell, w, \mathbf{x})}{t} \\
& =\lambda \frac{|\ell w-\ell x|}{t}=\lambda\left(\frac{1}{\mathbb{M}_{r}(\ell w, \ell x, t)}-1\right) .
\end{aligned}
$$

This implies that

$$
\frac{1}{\mathbb{M}_{r}(A w, B x, t)}-1 \leq \lambda\left(\frac{1}{\mathbb{M}_{r}(\ell w, \ell x, t)}-1\right) \text { for } t>0,
$$

for all $w, x \in C$. Thus, the operators $A, B$, and $\ell$ satisfy all the conditions of Corollary 16 with $\lambda=(a+b)$ in (57). Then, the operators $A, B$, and $\ell$ have a unique CFP $w^{*} \in C$, i.e., $w^{*}$ is a solution of the BVP (4.1).

(2) If $|\ell w-A w|$ is the maximum term in (77), then $\mathbf{M}(A, B, \ell, w, x)=|\ell w-A w|$. Now from (74) and (84), we have 


$$
\begin{aligned}
\frac{1}{\mathbb{M}_{r}(A w, B x, t)}-1 & =\frac{\mathfrak{D}(A w, B x)}{t} \leq \lambda \frac{\mathbf{M}(A, B, \ell, w, x)}{t} \\
& =\lambda \frac{|\ell w-A w|}{t}=\lambda\left(\frac{1}{\mathbb{M}_{r}(\ell w, A w, t)}-1\right) .
\end{aligned}
$$

This implies that

$\frac{1}{\mathbb{M}_{r}(A w, B x, t)}-1 \leq \lambda\left(\frac{1}{\mathbb{M}_{r}(\ell w, A w, t)}-1\right) \forall w, x \in C$ and $t>0$.

Similarly, if $|\ell x-B x|$ is the maximum term in (77), then, $\mathbf{M}(A, B, \ell, w, x)=|\ell x-B x|$. Now, by using (74) and (84), we get that

$$
\frac{1}{\mathbb{M}_{r}(A w, B x, t)}-1 \leq \lambda\left(\frac{1}{\mathbb{M}_{r}(\ell x, B x, t)}-1\right) \forall w, x \in C \text { and } t>0 .
$$

Again, if $|\ell x-A w|$ is the maximum term in (77), then, $\mathbf{M}(A, B, \ell, w, x)=|\ell x-A w|$. Now, by using (74) and (84), we get that

$\frac{1}{\mathbb{M}_{r}(A w, B x, t)}-1 \leq \lambda\left(\frac{1}{\mathbb{M}_{r}(\ell x, A w, t)}-1\right) \forall w, x \in C$ and $t>0$.

Next, if $|\ell w-B x|$ is the maximum term in (77), then, $\mathbf{M}(A, B, \ell, w, x)=|\ell w-B x|$. Now by using (74) and (84), we get that

$$
\frac{1}{\mathbb{M}_{r}(A w, B x, t)}-1 \leq \lambda\left(\frac{1}{\mathbb{M}_{r}(\ell w, B x, t)}-1\right) \forall w, x \in C \text { and } t>0 \text {. }
$$

Hence, from (88), (89), (90), and (91), the operators $A, B$, and $\ell$ satisfy all the conditions of Corollary 16 with $\lambda=b$ and $a=0$ in (57). Thus, the operators $A, B$, and $\ell$ have a unique CFP $w^{*} \in C$, i.e., $w^{*}$ is a solution of the BVP (70).

\section{Conclusion}

In this paper, we established the concept of rational type weakly-compatible fuzzy-contraction for three selfmappings in complete FM spaces and proved some coincidence point and CFP results under the rational type weakly-compatible fuzzy-contraction conditions by using the "triangular property of fuzzy metric" in complete FM spaces with examples. Further, as an application, we presented fuzzy differential equations and proved that the solution of the FDEs has a unique CFP of the integral operators, that is, $A, B$, and $\ell$. This new direction of rational type weakly-compatible fuzzy-contraction with the application of FDEs in FM space will play a very important role. This idea can be extended and generalized in different directions with different types of weakly-compatible fuzzy-contraction conditions for self-mappings with different types of differential equations in the context of FM spaces.

\section{Data Availability}

Data sharing is not applicable to this article as no data set were generated or analysed during the current study.

\section{Conflicts of Interest}

The authors declare that there is no conflict of interest regarding the publication of this paper.

\section{Acknowledgments}

The authors are grateful to the Deanship of Scientific Research, King Saud University for funding through Vice Deanship of Scientific Research Chairs.

\section{References}

[1] S. Banach, "Sur les opérations dans les ensembles abstraits et leur application aux équations intégrales," Fundamenta Mathematicae, vol. 3, pp. 133-181, 1922.

[2] R. Kannan, "Some results on fixed points," Bulletin of the Calcutta Mathematical Society, vol. 60, pp. 71-76, 1968.

[3] S. K. Chatterjea, "Fixed point theorems," Dokladi na B"lgarskata Akademiya na Naukite, vol. 25, pp. 727-730, 1972.

[4] D. Chatterjea, "Generalized contraction principle," International Journal of Mathematics and Mathematical Sciences, vol. 6, no. 1, 94 pages, 1983.

[5] M. U. Ali, H. Aydi, and M. Alansari, "New generalizations of set valued interpolative Hardy-Rogers type contractions in bmetric spaces," Journal of Function Spaces, vol. 2021, Article ID 6641342, 8 pages, 2021.

[6] H. Covitz and S. B. Nadler, "Multi-valued contraction mappings in generalized metric spaces," Israel Journal of Mathematics, vol. 8, no. 1, pp. 5-11, 1970.

[7] I. Altun, M. Aslantas, and H. Sahin, "KW-type nonlinear contractions and their best proximity points," Numerical Functional Analysis and Optimization, vol. 42, no. 8, pp. 935-954, 2021.

[8] A. R. Khan, "Properties of fixed point set of a multivalued map," Journal of Applied Mathematics and Stochastic Analysis, vol. 2005, no. 3, pp. 323-331, 2005.

[9] S. U. Rehman, S. Jabeen, and H. U. H. Muhammad, "Some multi-valued contraction theorems on H-cone metric," Journal of Advanced Studies in Topology, vol. 10, no. 2, pp. 1124, 2019.

[10] H. Sahin, "Best proximity point theory on vector metric spaces," Communications Faculty Of Science University of Ankara Series A1Mathematics and Statistics, vol. 70, no. 1, pp. 130-142, 2021.

[11] L. A. Zadeh, "Fuzzy sets," Information and control, vol. 8, no. 3, pp. 338-353, 1965.

[12] O. Kramosil and J. Michalek, "Fuzzy metric and statistical metric spaces," Kybernetika, vol. 11, pp. 336-344, 1975.

[13] M. Grabiec, "Fixed points in fuzzy metric spaces," Fuzzy Sets and Systems, vol. 27, no. 3, pp. 385-389, 1988. 
[14] A. George and P. Veeramani, "On some results in fuzzy metric spaces,” Fuzzy Sets and Systems, vol. 64, no. 3, pp. 395-399, 1994.

[15] V. Gregori and A. Sapena, "On fixed-point theorems in fuzzy metric spaces," Fuzzy Sets and Systems, vol. 125, no. 2, pp. 245-252, 2002.

[16] Z. Sadeghi, S. M. Vaezpour, C. Park, R. Saadati, and C. Vetro, "Set-valued mappings in partially ordered fuzzy metric spaces," Journal of Inequalities and Applications, vol. 2014, no. 1, 2014.

[17] C. D. Bari and C. Vetro, "Fixed points, attractors and weak fuzzy contractive mappings in a fuzzy metric space," Journal of Fuzzy Mathematics, vol. 1, pp. 973-982, 2005.

[18] O. Hadzic and E. Pap, "A fixed point theorem for multivalued mappings in probabilistic metric spaces and an application in fuzzy metric spaces," Fuzzy Sets and Systems, vol. 127, no. 3, pp. 333-344, 2002.

[19] M. Imdad and J. Ali, "Some common fixed point theorems in fuzzy metric spaces," Mathematical Communications, vol. 11, pp. 153-163, 2006.

[20] I. Shamas, S. U. Rehman, H. Aydi, T. Mahmood, and E. Ameer, "Unique fixed-point results in fuzzy metric spaces with an application to Fredholm integral equations," Journal of Function Spaces, vol. 2021, Article ID 4429173, 12 pages, 2021.

[21] T. Som, "Some results on common fixed point in fuzzy metric spaces," Soochow Journal of Mathematics, vol. 33, pp. 553-561, 2007.

[22] B. D. Pant and S. Chauhan, "Common fixed point theorems for two pairs of weakly compatible mappings in menger spaces and fuzzy metric spaces," Scientific Studies and Research, vol. 21, pp. 81-96, 2011.

[23] H. N. Saleh, M. Imdad, and M. Hasanuzzaman, "A short and sharpened way to approach fixed point results involving fuzzy $\mathscr{H}$-contractive mappings," Fixed Point Theory and Applications, vol. 2020, no. 1, 2020.

[24] H. N. Saleh, M. Imdad, I. Khan, and M. Hasanuzzaman, "Fuzzy $\Theta f$-contractive mappings and their fixed points with applications," Journal of Intelligent \& Fuzzy Systems, vol. 39, no. 5, pp. 7097-7106, 2020.

[25] S. U. Rehman, R. Chinram, and C. Boonpok, "Rational type fuzzy-contraction results in fuzzy metric spaces with an application," Journal of Mathematics, vol. 2021, Article ID 6644491, 13 pages, 2021.

[26] T. Oner, M. B. Kandemir, and B. Tanay, "Fuzzy cone metric spaces," Journal of Nonlinear Sciences and Applications, vol. 8, no. 5, pp. 610-616, 2015.

[27] S. U. Rehman and H.-X. Li, "Fixed point theorems in fuzzy cone metric spaces," The Journal of Nonlinear Sciences and Applications, vol. 10, no. 11, pp. 5763-5769, 2017.

[28] S. Jabeen, S. U. Rehman, Z. Zheng, and W. Wei, "Weakly compatible and quasi-contraction results in fuzzy cone metric spaces with application to the Urysohn type integral equations," Advances in Differential Equations, vol. 2020, no. 1, 2020.

[29] S. U. Rehman and H. Aydi, "Rational fuzzy cone contractions on fuzzy cone metric spaces with an application to Fredholm integral equations," Journal of Function Spaces, vol. 2021, Article ID 5527864, 13 pages, 2021.

[30] B. Schweizer and A. Sklar, "Statical metric spaces," Pacific Journal of Mathematics, vol. 10, pp. 314-334, 1960.
[31] M. Abbas and G. Jungck, "Common fixed point results for noncommuting mappings without continuity in cone metric spaces," Journal of Mathematical Analysis and Applications, vol. 341, no. 1, pp. 416-420, 2008.

[32] V. Lakshmikantham and R. Mohapatra, Theory of Fuzzy Differential Equations and Inclusion, Taylor and Francis, London, 2004. 